\title{
Conservative Management of Spinal Tuberculosis: Initial Series from Pakistan
}

\author{
Asad Abbas, Syed Raza Haider Rizvi, Mufaddal Mahesri, Hisham Raza Aleem Salahuddin \\ Department of Surgery, Ziauddin University Hospital, Karachi, Pakistan
}

\begin{abstract}
Study Design: A prospective study on spinal tuberculosis (TB) at a tertiary care hospital in an endemic region.
Purpose: The aim of the study is to reiterate the importance of conservative management of spinal TB.

Overview of Literature: Spinal tuberculosis can present with wide spectrum of symptoms, with back pain being the most common symptom. It is the leading cause of non-traumatic paraplegia in developing countries. There is an emerging trend to operate on patients early with spinal TB.

Methods: Forty-seven (M=14, F=33) patients were enrolled in the study during the four year study period. Initially, all the patients were subjected to computed tomography guided percutaneous needle aspiration (PCNA) followed by antituberculous therapy (ATT) for 12 months. Indications for surgery included patients with moderate to severe symptoms in which PCNA either failed, was impossible to carry out, or produced minimal improvement within 48 hours.

Results: Presenting complaints included pain (95.7\%), weakness (85.1\%) and sphincter involvement (12.8\%). On the magnetic resonance imaging, a paravertebral abscess was seen in $37(78.7 \%)$, disc and body destruction in $29(61.7 \%)$, and an epidural abscess in $12(25.9 \%)$ patients. Of the 47 patients, $9(19.1 \%)$ required surgery, 4 of whom had failed PCNA attempts and 5 demonstrated indications despite successful PCNA.

Conclusions: The results of conservative treatment consisting of PCNA and ATT for at least 12 months in compliant patients are excellent. A combined approach using clinical staging, PCNA, and ATT can minimize surgical intervention in most patients. However, ATT remains to be the cornerstone of management of spinal TB.
\end{abstract}

Keywords: Spinal tuberculosis; Fine needle aspiration; Surgical decompression; Anti-tuberculosis

\section{Introduction}

Tuberculosis (TB) is a rampant disease with 8.8 million new cases reported in 2010 [1]. According to the World Health Organization (WHO) it is the leading infectious cause of mortality worldwide killing 1.45 million people [1]. Although the majority of the cases of TB occur in developing countries, recently an alarming increase in the incidence of TB has been reported in many of the devel- oped countries. This resurgence of TB is being attributed to the increased incidence of alcohol and drug abuse, human immunodeficiency virus, immunosuppression [2], and the increased influx of immigrants from third-world countries [3].

Extrapulmonary manifestations of TB are also fairly common with skeletal system involvement being the most frequent [4]. Spinal tuberculosis, first described by Sir Percival Pott in the Eighteenth century, accounts for 50\%

Received Mar 20, 2012; Revised May 13, 2012; Accepted May 14, 2012

Corresponding author: Asad Abbas

Department of Surgery, Ziauddin University Hospital, ST-4/B, Shahrah-e-Ghalib, Clifton Block-6, Karachi, Pakistan

Tel: +92-322-251-7014, Fax: +92-21-3586-2940, E-mail: asad.abbas@live.com

*2010 NSA Annual Scientific Meeting, September 30 - October 2, 2010, Sunshine Coast, Queensland, Australia. 
of these cases [5] and results in immense morbidity and mortality. Although spinal TB presents clinically with a multitude of symptoms, back pain is the major symptom. Spinal TB can lead to bone destruction and vertebral collapse resulting in paravertebral abscesses and deformity. If prompt management is not employed, severe neurological symptoms ensue, which can lead to a debilitating consequence. In fact, spinal TB still remains the leading cause of non-traumatic paraplegia in developing nations $[6,7]$.

If cases of spinal TB are diagnosed early, anti-tubercular chemotherapy along with immobilization can be effective. It has been argued that if patients do not respond to conservative treatment or show progressive neurological symptoms, spinal deformity or instability, surgical intervention should be the mainstay of treatment $[8,9]$. However, anterior resection or combined anterior and posterior resection can result in substantial morbidity [10]. As a result, percutaneous needle aspiration (PCNA) to drain tuberculous abscesses has gained increasing popularity in the management of spinal TB $[4,11]$. The use of PCNA along with antituberculous drug therapy has shown encouraging results and seems to be an effective prospect in the management of spinal TB.

In this prospective study, we present our experience of conservative management of spinal TB from an endemic region, Pakistan. This is to reiterate the importance of managing spinal tuberculosis conservatively, keeping surgical intervention as the last resort.

\section{Materials and Methods}

\section{Patients}

During the four year study period from January 2006 to December 2009, 47 patients ( $M=14, F=33)$ attending Ziauddin University Hospital were included in the study. Prior to enrollment, an informed consent was taken from all patients. Tuberculosis of the spine was suspected on the basis of history followed by an X-ray (Fig. 1) and a magnetic resonance imaging (MRI) of the spine. In our study, suspected MRI lesions were those which showed an isointense lesion on $\mathrm{T} 1$ or a high signal lesion on T2 involving the disc, body, paravertebral region and sometimes extending into the epidural space or psoas fascia (Figs. 2, 3). The patients were divided into either mild, or a moderate to severe group, on the basis of symptoms and

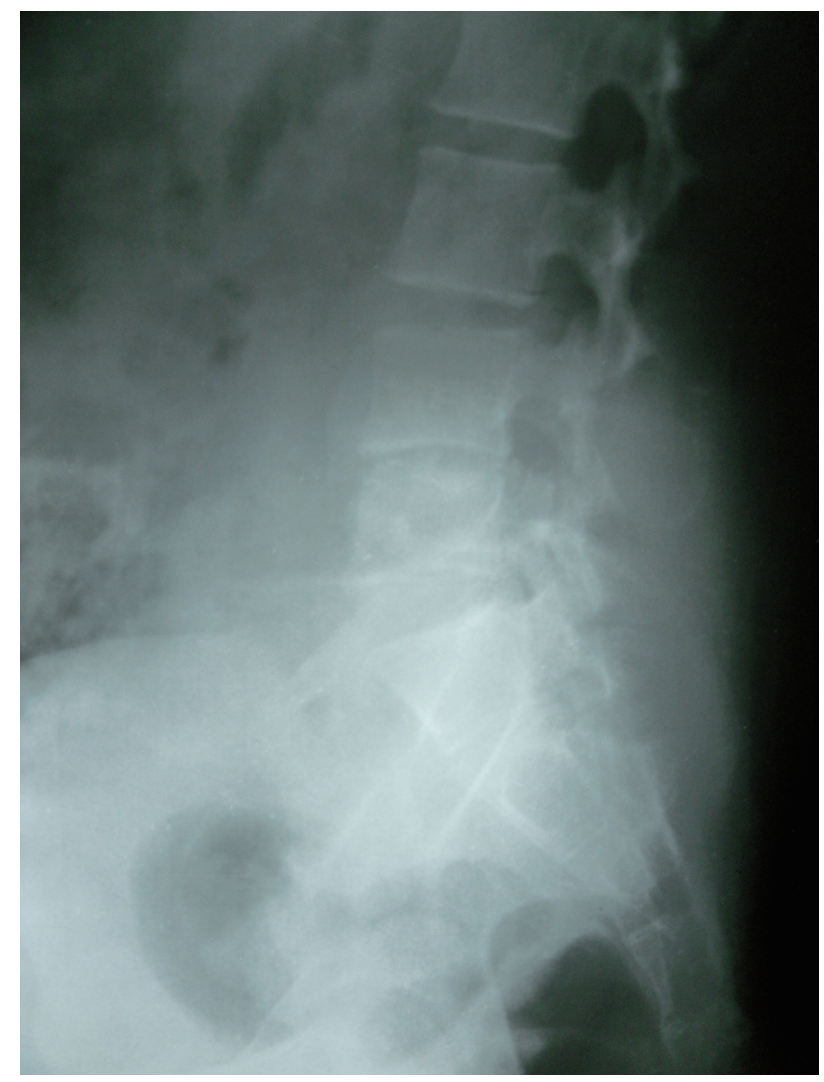

Fig. 1. X-ray of the lumbosacral spine lateral view showing sclerotic change and minimal erosion of the upper end plate of $L 5$ vertebra. There is reduced disc space at $L 4-5$.

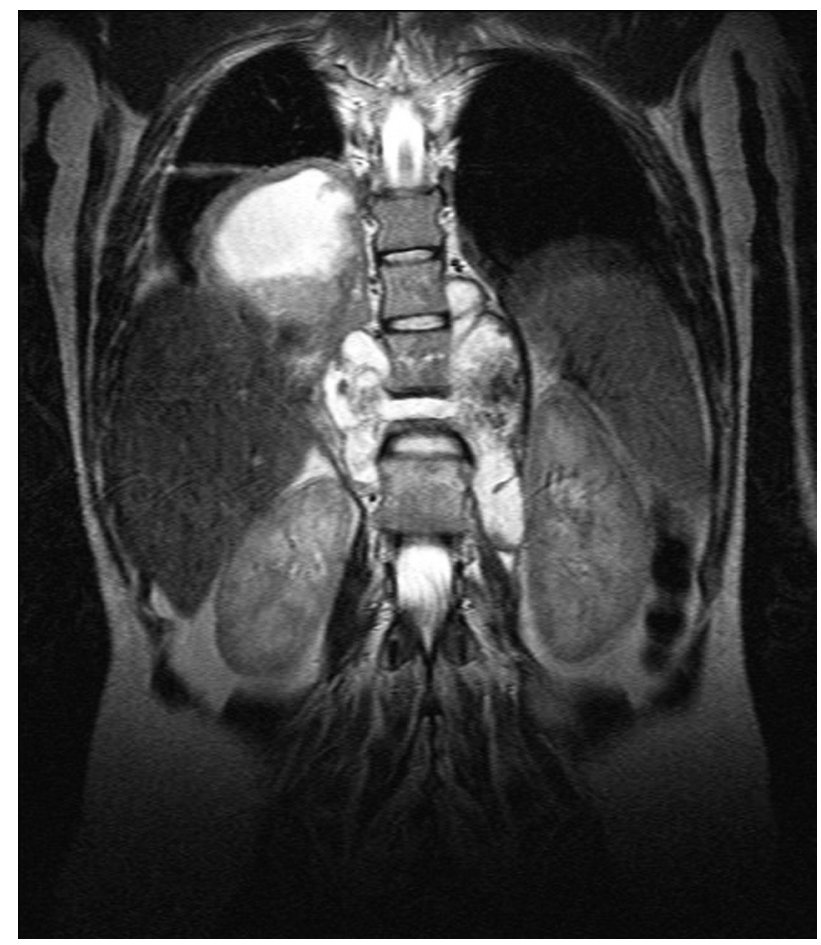

Fig. 2. T2 weighted magnetic resonance imaging showing a bilateral paravertebral and psoas abscess, the abscess is also extending towards the right hemithorax elevating the dome of the diaphragm. 
signs. Mild patients were classified as those having any degree of pain and/or mild motor weakness. Moderatesevere patients had severe motor deficits or any degree of sphincter disturbance. All patients were initially subject to computed tomography (CT) guided PCNA for the confirmation of diagnosis. Simultaneously, antituberculous therapy (ATT) was also started and continued for at least 12 months (average, 12.3 months; maximum, 18 months) considering the endemic state of TB in this region.

Patients with mild symptoms were kept on ATT for the defined period irrespective of the outcome of PCNA. Patients with moderate-severe symptoms who responded to successful PCNA in terms of significant improvement of symptoms especially pain within 48 hours of the procedure were kept on ATT only.

\section{Anti-tuberculous therapy}

The standard ATT consisted of four drugs; isoniazid (5 mg/kg; maximum, $300 \mathrm{mg} /$ day), rifampicin $15 \mathrm{mg} /$ $\mathrm{kg}$; maximum, $600 \mathrm{mg} /$ day, ethambutol (15-25 mg/kg; maximum, $2 \mathrm{~g} /$ day), pyrazinamide $(15-30 \mathrm{mg} / \mathrm{kg}$; maxi- mum, $2 \mathrm{~g}$ /day). Pyridoxine was also added to the regimen prophylactically. This regimen was instituted for three months followed by withdrawal of pyrazinamide for the rest of the period.

\section{Percutaneous needle aspiration}

PCNA was performed using CT guidance, for which patients were placed in a prone or lateral position on the CT scanner table. Few pilot scans were taken beforehand to tailor the approach for safe insertion of the spinal needle and localization of the lesion. An 18 gauge spinal needle was inserted percutaneously, depth and route of which was dictated by CT scan. Necrotic material was aspirated as much as possible using a $10 \mathrm{~mL}$ syringe. If blocked, the needle was replaced with another needle until the aspirate stopped coming. The sample collected from PCNA was sent for an acid fast bacillus (AFB) smear as well as culture and sensitivity of Mycobacterium tuberculosis.

\section{Surgery}

Patients with moderate-severe disease who did not show
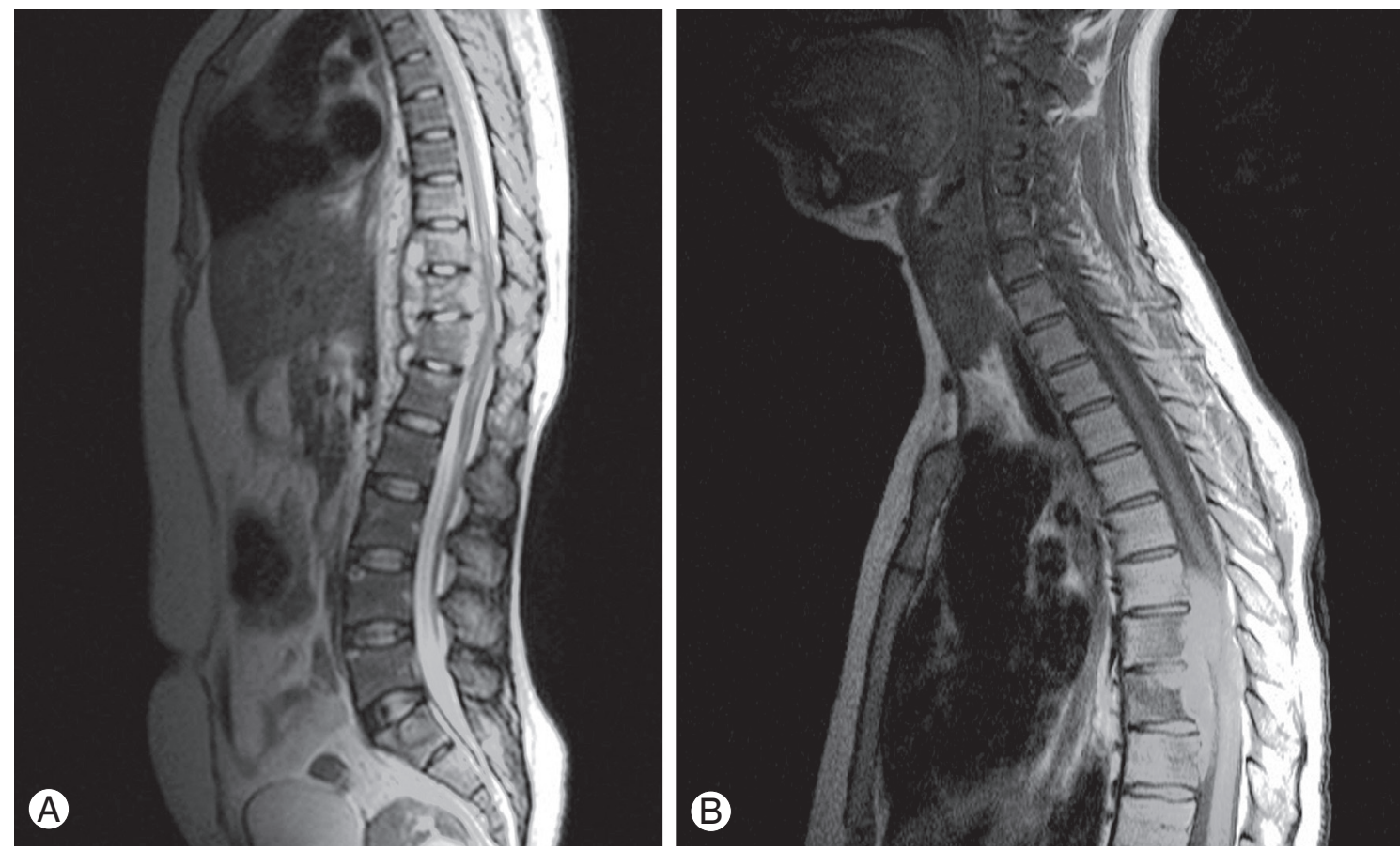

Fig. 3. (A) T2 weighted magnetic resonance imaging (MRI) showing an altered signal intensity lesion involving D10 to D12 vertebral bodies with Paravertebral soft tissue collection encroaching over the spinal cord. This is an atypical presentation sparing the disc spaces. The diagnosis was later confirmed on percutaneous needle aspiration (PCNA) as Tuberculosis. (B) T1 weighted MRI showing involvement of the posterior elements of D8 to D10 vertebral bodies, an unusual presentation of PCNA confirms Tuberculosis. An epidural collection is also seen. 
remarkable improvements within 48 hours after successful PCNA were candidates for surgery. These patients showed persistent symptoms despite PCNA and ATT. Patients in the same severity group with failed PCNA were also enrolled for operative management. This group did not respond to ATT alone and had persistent neurology. Spinal deformity or instability was not the indication for surgery in any of our patients.

The surgical procedure varied from anterio-lateral to posterior decompression. The prime objective of surgical procedure was to debride the necrotic material causing the pressure effect on the thecal sac while secondarily keeping removal of bone to a minimum. This resulted in no significant increase in instability or deformity with regards to the spine thus the need for instrumentation was eliminated. Moreover, all patients who underwent surgery for the cervical, the thoraco-lumbar junction or the lumbar region were prescribed an appropriate brace or collar for 6 months postoperatively.

\section{Follow-up}

Patients were recalled at 6 week intervals for the first two visits followed by 3 monthly reviews until 12 months after the end of ATT. The mean follow-up period was 24.5 months (range, 24-36 months). They were managed and monitored for pain, erythrocyte sedimentation rate (ESR), and neurological status with special attention given to motor weakness. Follow up X-rays of the spine were done at 3 monthly intervals, or in those who could afford it, MRIs were completed at 6 monthly intervals. Imaging studies to monitor abscess recurrence were completed regularly until end of ATT treatment with a last imaging study 6 months after the completion of ATT. Smoothening of the vertebral end plates with or without fusion of vertebral bodies on X-ray (Fig. 4) was considered as evidence of healing. Moreover, loss of high signal on T2 weighted MRI along with resorption of abscess (Fig. 5) further confirmed the resolution of infection.

\section{Results}

During the four year study period 47 patients $(\mathrm{M}: \mathrm{F}=1: 2.35)$ attending our center were included in the study. The mean age of individuals was $33.9 \pm 13.7$ years. Presenting complaints included pain in 45 (95.7\%), motor weakness in $40(85.1 \%)$ and sphincter involvement in 6

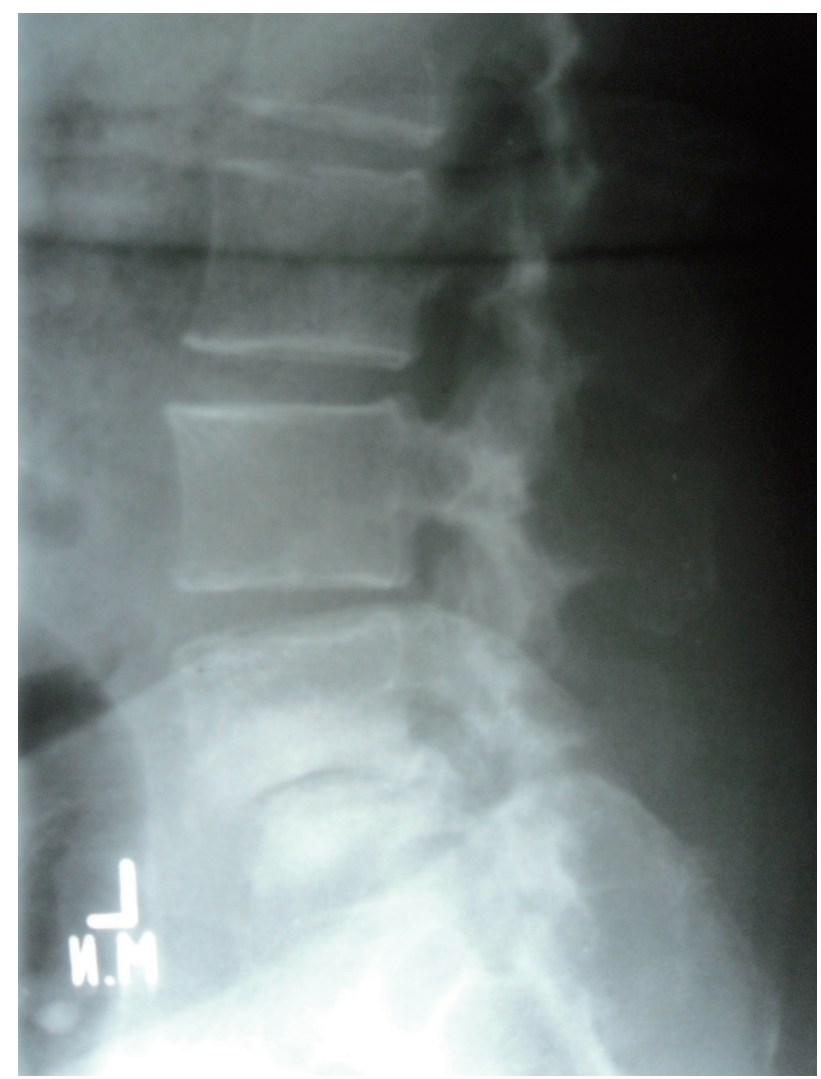

Fig. 4. X-ray lumbosacral spine at the end of 12 months of antituberculous therapy showing reduced disc space at L4-L5 associated with reduced $L 5$ vertebral body height.

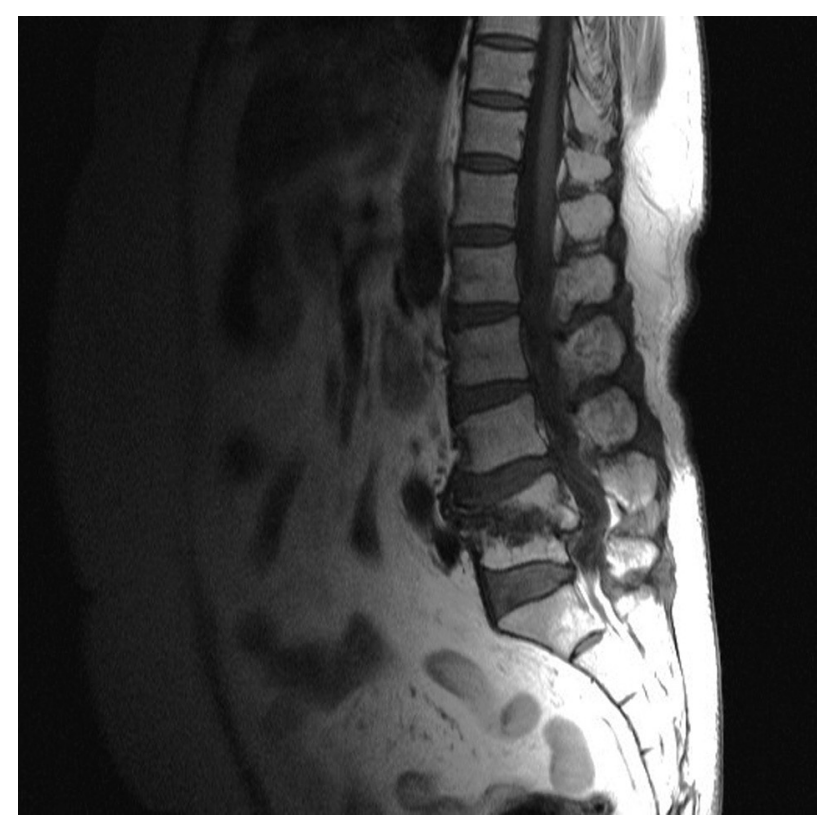

Fig. 5. T1 weighted magnetic resonance imaging showing healed Ttuberculosis of the spine for L4-L5 after 12 months of antituberculous therapy. 
(12.8\%) patients. An MRI of the spine showed a paravertebral abscess in 37 (78.7\%), disc and body destruction in $29(61.7 \%)$ and an epidural abscess in $12(25.5 \%)$ patients whereas, psoas abscess was seen in $4(8.5 \%)$ patients. Two (4.2\%) patients had concomitant hydrocephalous secondary to TB meningitis at the time of presentation and hence, were candidates for a ventriculoperitoneal (VP) shunt and extended ATT treatment up to 18 months.

Regarding the grade of motor weakness, 2 (5\%) had power 5/5, 22 (55\%) had 4/5, 14 (35\%) had 3/5, 1 (2.5\%) had $2 / 5$, and $1(2.5 \%)$ had power $1 / 5$ graded on the basis of the patient's effort [12] at presentation.

The cervical spine was involved in $6(12.8 \%)$, the cervico-dorsal spine in $1(2.1 \%)$, the dorsal spine in $16(34 \%)$, the dorso-lumbar spine in 4 (8.5\%), the lumbar spine in $19(40.4 \%)$ and the lumbo-sacral spine was involved in $1(2.1 \%)$ patient. Ten $(21.2 \%)$ patients had single level involvement, 35 (74.4\%) patients have two levels, and 2 (4.2\%) patients showed three or more levels of involvement. L1 was the most common level involved in our study with $10(21.2 \%)$ patients affected at this level of the spine, irrespective of the number of levels and region of spine involved.

All patients were initially planned for PCNA; however four patients denied undergoing PCNA and hence, were continued on ATT only. PCNA was successfully performed on 39 (90.6\%) patients while it failed in 4 (9.3\%) patients. All patients underwent single aspiration except two. The first patient in this series had PCNA done in two sittings, which was done electively because of a large amount of bilateral abscesses. Another patient required repeat PCNA treatment one month later during the treatment phase due to a residual abscess. The total amount of necrotic material aspirated during successful PCNA varied from $0.5 \mathrm{~mL}$ to as much as $300 \mathrm{~mL}$ with a mean of $12.2 \mathrm{~mL}$. Following successful PCNA, AFB smear was positive in 35 (89.7\%) patients while the cultures turned out to be positive in all cases. All cultured bacilli were sensitive to first line anti-tuberculous drugs.

The mean ESR during presentation was $64.2 \pm 6.3 \mathrm{~mm}$ in the first hour, which fell to $37.3 \pm 3.85 \mathrm{~mm}$ in the first hour at 6 weeks on ATT. At the end of treatment, mean ESR was $14.2 \pm 2.6 \mathrm{~mm}$, which corresponded with resolution of symptoms.

Decompressive surgery was performed in $9(19.1 \%)$ patients, with 7 (14.9\%) undergoing anterio-lateral decompression and 2 (4.2\%) undergoing posterior decompres- sion; however, instrumentation was not performed on any of our patients for the aforementioned reason. Out of these surgical candidates, 4 had failed PCNA attempts and 5 had indications despite successful PCNA including persistent motor weakness, pain and/or sphincter involvement. All surgical patients were prescribed appropriate spinal braces for 6 months postoperatively, in order to provide external support to the spine since instrumentation was avoided.

Forty-four (93.6\%) patients in our study showed good compliance with ATT therapy and fully recovered without any residual weakness or pain. However, 3 (6.4\%) patients were non-compliant with the treatment. Patients who demonstrated poor compliance deteriorated during the course. One compliant patient died of an unrelated medical condition before the completion of the regimen. Moreover, none of the patients showed any signs for the recurrence of $\mathrm{TB}$, in the spine or any other region of the body, during the follow-up period.

\section{Discussion}

According to the Global Tuberculosis Control WHO Report 2011 [1], Pakistan is amongst the highest burdened countries for tuberculosis with 150 per 100,000 new cases in 2010. This high incidence is due to a number of factors including low socio-economic conditions, over-crowding, multi-drug resistance and most importantly, non-compliance to ATT.

The mean age at presentation in our study was 33.9 years with a range of 12 to 68 years which is about a decade younger than that found in other studies [4]. An overwhelming number of patients in our study were female ( $M: F=1: 2.35$ ). This is in contrast to the extensively reported male predominance within the literature $[3,8,13]$. However, another study from the same region reported similar trends for younger individuals and gender predominance [14].

In our study the most common symptoms presented were pain and motor weakness. Axial pain of insidious onset is regarded as one of the most common symptoms of spinal tuberculosis. However, it can present itself with a wide range of symptoms and complications $[15,16]$. Atypical presentations for TB of the spine include destruction of the posterior arch, isolated neural arch or disease without disc involvement (Fig. 3). Other clinical features in the active stage of the disease are malaise, 
weight loss, anorexia, night sweats and low grade fever with stiff spine and sometimes kyphosis or cold spine [17]. This wide range of clinical presentations makes the diagnosis of spinal TB difficult.

The diagnosis can be made using an AFB smear, an AFB culture and the polymerase chain reaction (PCR) of Mycobacterium tuberculosis. The ESR and Mantoux test can be used to assist in the diagnosis. Radiological studies also play a key role in establishing the diagnosis of spinal TB.

The optimal technique for the diagnosis of a spinal infection and its sequel is MRI [18]. The difference between inflammatory and neoplastic pathologies may not be appreciated on plain X-rays or CT scans and hence, MRI is needed, which reveals $\mathrm{TB}$ as a contrast enhancing rim [19]. This appearance can be readily differentiated from a metastatic lesion first involving the pedicles, which are initially spared in spinal TB. In addition to MRI, a CTguided biopsy has been regarded as an efficacious method for the establishment of diagnosis [11,20]. Finally, adequate response to ATT can also be used to establish the diagnosis [21].

The AFB smear was prepared using Ziehl Neelsen stain and Lowenstein-Jensen medium was used for the culture of the organism. Although PCR is a quicker and more reliable way for detecting tuberculous bacillus, it was not used in our study considering its high cost and extensive positivity of the AFB smear and culture in our patients.

It has been reported that the dorsal spine is the most common site of involvement followed by the lumbar, the dorso-lumbar, and the cervical spine [14,22]. However, in our study the dorsal spine was the second most frequent region involved following the lumbar spine, with L1 being the most frequently affected level irrespective of the number of levels involved. Tuberculosis frequently involves multiple spinal levels; as many as 17 vertebrae have been reported to be involved [14].

The success rate of PCNA in our study was $90.6 \%$, which yielded a sufficient sample for the AFB and the culture. However, it has been reported that the sample is often inadequate [4]. We had a positive AFB smear in $89.7 \%$ and a positive culture in all successful PCNAs. However, a positive culture of $76 \%$ has been reported by Staatz et al. [23] using CT-guided PCNA. CT-guided PCNA has been shown to be successful in draining almost all abscesses from the neck to the sacroiliac joint $[4,24]$.
In our study only $9(19.1 \%)$ patients underwent surgery; 4 of which had failed PCNA and 5 had persistent symptoms. Indications for surgery include failed conservative management, spinal deformity or instability, and/ or persistent neurological symptoms; however, this surgical approach has a $2 \%$ to $11 \%$ mortality rate and a recurrence rate of $10 \%$ to $20 \%$ [25].

It has been claimed that patients with a neurological deficit show better recovery with surgery and chemotherapy as opposed to those on chemotherapy only [20]. According to the fourteenth report of the Medical Research Council Working Party regarding Tuberculosis of the Spine, surgical debridement had no significant therapeutic advantage over chemotherapy alone. Additionally, PCNA reduces damage to adjacent structures and also eliminates the risk of general anesthesia [24,26]. Lastly, perioperative morbidity and mortality should not be overlooked.

Of the 47 patients, $93.6 \%(n=44)$ demonstrated good compliance with the treatment regimen and showed complete recovery at the end of treatment. All 3 patients who exhibited poor compliance deteriorated steadily and eventually stopped attending the clinic, furthermore they did not respond to hospital queries. This reiterates the importance of compliance with ATT. Additionally, Directly Observed Treatment Strategy can be used not only to improve compliance but also to prevent the development of multi-drug resistant forms of TB [1].

\section{Limitations}

The study was conducted on patients presenting to a tertiary care urban hospital. The sample does not include patients from rural areas of the country where TB spine is diagnosed at a much advanced stage. Most of our patients presented with symptoms of early stages of the illness without any gross deformity or instability. They were diagnosed in a timely fashion due to the high index of suspicion within the endemic region. Therefore, the results may not be generalized to regions where spinal TB is rare.

\section{Conclusions}

A combined approach using clinical staging, PCNA, and ATT can minimize surgical intervention in most patients and thus avoid potential complications as well as further instability associated with it. The results of the conserva- 
tive treatment consisting of PCNA and ATT for at least 12 months in compliant patients are excellent. Early diagnosis and treatment before the development of spinal deformity seems to be essential for better outcomes and survival. However, ATT remains to be the cornerstone of management with regards to spinal TB.

\section{Conflict of Interest}

No potential conflict of interest relevant to this article was reported.

\section{Acknowledgements}

Dr. Anwar Ahmed and Dr. Irfan Lutfi, Department of Radiology, Ziauddin University, for their cooperation with PCNA and Radiological imaging during the study.

\section{References}

1. World Health Organization Global tuberculosis control: WHO report 2011 [Internet]. Geneva: World Health Organization; 2011 [cited 2013 March 15]. Available from http://www.who.int/tb/publications/ global_report/2011/gtbr11_full.pdf.

2. Nelson CA, Zunt JR. Tuberculosis of the central nervous system in immunocompromised patients: HIV infection and solid organ transplant recipients. Clin Infect Dis 2011;53:915-26.

3. Kamper-Jorgensen Z, Andersen AB, Kok-Jensen A, et al. Migrant tuberculosis: the extent of transmission in a low burden country. BMC Infect Dis 2012;12:60.

4. Dinc H, Ahmetoglu A, Baykal S, Sari A, Sayil O, Gumele HR. Image-guided percutaneous drainage of tuberculous iliopsoas and spondylodiskitic abscesses: midterm results. Radiology 2002;225:353-8.

5. Buxi TB, Sud S, Vohra R. CT and MRI in the diagnosis of tuberculosis. Indian J Pediatr 2002;69:965-72.

6. Rajasekaran S, Prasad Shetty A, Dheenadhayalan J, Shashidhar Reddy J, Naresh-Babu J, Kishen T. Morphological changes during growth in healed childhood spinal tuberculosis: a 15-year prospective study of 61 children treated with ambulatory chemotherapy. J Pediatr Orthop 2006;26:716-24.

7. Teo HE, Peh WC. Skeletal tuberculosis in children. Pediatr Radiol 2004;34:853-60.

8. Garg B, Kandwal P, Nagaraja UB, Goswami A, Jayas- wal A. Anterior versus posterior procedure for surgical treatment of thoracolumbar tuberculosis: a retrospective analysis. Indian J Orthop 2012;46:165-70.

9. Oguz E, Sehirlioglu A, Altinmakas M, et al. A new classification and guide for surgical treatment of spinal tuberculosis. Int Orthop 2008;32:127-33.

10. Yang SC, Fu TS, Chen LH, Niu CC, Lai PL, Chen WJ. Percutaneous endoscopic discectomy and drainage for infectious spondylitis. Int Orthop 2007;31:367-73.

11. Francis IM, Das DK, Luthra UK, Sheikh Z, Sheikh M, Bashir M. Value of radiologically guided fine needle aspiration cytology (FNAC) in the diagnosis of spinal tuberculosis: a study of 29 cases. Cytopathology 1999;10:390-401.

12. Medical criteria.com. Medical research council (MRC) scale for muscle strength [Internet]. 2009 [cited 2012 Apr 7]. Available from: http://www.medicalcriteria.com/site/index.php?option $=$ com_conten $\mathrm{t} \&$ view $=$ article\&id=238\%3Aneuromrc\&catid $=64 \%$ $3 \mathrm{~A}$.

13. N’Dri Oka D, N'Dri-Yoboue MA, Varlet G, Haidara A, Ba Zeze V. Spinal tuberculosis. Epidemiologic and diagnostic aspects: a study of 28 clinical observations. Sante 2004;14:81-4.

14. Bakhsh A. Medical management of spinal tuberculosis: an experience from Pakistan. Spine (Phila Pa 1976) 2010;35:E787-91.

15. Belzunegui J, Intxausti JJ, De Dios JR, et al. Haematogenous vertebral osteomyelitis in the elderly. Clin Rheumatol 2000;19:344-7.

16. Gasbarrini AL, Bertoldi E, Mazzetti M, et al. Clinical features, diagnostic and therapeutic approaches to haematogenous vertebral osteomyelitis. Eur Rev Med Pharmacol Sci 2005;9:53-66.

17. Kumar R. Spinal tuberculosis: with reference to the children of northern India. Childs Nerv Syst 2005;21:19-26.

18. Forrester DM. Infectious spondylitis. Semin Ultrasound CT MR 2004;25:461-73.

19. Umredkar A, Mohindra S, Chhabra R, Gupta R. Vertebral body hyperostosis as a presentation of Pott's disease: a report of two cases and literature review. Neurol India 2010;58:125-7.

20. Boachie-Adjei O, Squillante RG. Tuberculosis of the spine. Orthop Clin North Am 1996;27:95-103.

21. Krishnan A, Patkar D, Patankar T, et al. Craniovertebral junction tuberculosis: a review of 29 cases. J 
Comput Assist Tomogr 2001;25:171-6.

22. Alothman A, Memish ZA, Awada A, et al. Tuberculous spondylitis: analysis of 69 cases from Saudi Arabia. Spine (Phila Pa 1976) 2001;26:E565-70.

23. Staatz G, Adam GB, Keulers P, Vorwerk D, Gunther RW. Spondylodiskitic abscesses: CT-guided percutaneous catheter drainage. Radiology 1998;208:363-7.

24. Zhang XF, Wang Y, Liu ZS, et al. Percutaneous focal debridement and local chemotherapy in the treat- ment of spinal tuberculous abscesses (Chin). Chin J Spine Spine Cord 2005;15:528-30.

25. Rothman SL. The diagnosis of infections of the spine by modern imaging techniques. Orthop Clin North Am 1996;27:15-31.

26. Muzii VF, Mariottini A, Zalaffi A, Carangelo BR, Palma L. Cervical spine epidural abscess: experience with microsurgical treatment in eight cases. J Neurosurg Spine 2006;5:392-7. 